\title{
Developing an Ambient Intelligent-Based Decision Support System for Production and Control Planning
}

\author{
Marco Gomes, Fábio Silva, Filipa Ferraz, António Silva, Cesar Analide and Paulo \\ Novais \\ Department of Informatics, University of Minho, Braga, Portugal \\ \{marcogomes, fabiosilva, analide,pjon\}@di.uminho.pt, \\ filipatferraz@gmail.com, antoniosilva9116@gmail.com
}

\begin{abstract}
Scheduling production instructions in a manufacturing facility is key to assure a efficient process that assures the desired product quantities are produced in time, with quality and with the right resources. An efficient production avoids the creation of downstream delays, and early completion which both can be detrimental if storage space is limited and contracted quantities are important. Therefore, the production, planning and control of manufacturing is increasingly more difficult as family products increases. This paper presents an ongoing Ambient Intelligent decision support system development that aims to provide assistance on the creation on standard work procedures that assure production quantity and efficiency by means of ambient intelligence, optimization heuristics and machine learning in the context of a large organization.
\end{abstract}

Keywords: Ambient Intelligence, Industry 4.0, Standard Work Decision Support System

\section{Introduction}

This project fits in a strategic partnership between our a research laboratory and a large Portuguese manufacture factory, which the main business is the development and production of infotainment and instrumentation systems for the automotive industry. These synergies were promoted to answer the challenge of improving the existent methodologies in order to increase the competitiveness, efficiency and productivity through systematic innovation.

Involved in such initiative, the work portrayed in this document outlines the phase of requirements specification, adaptation of ambient intelligence in the context of industry 4.0 and elaboration of intelligent software architectures. The main objective is to answer each requirement from the stakeholder of the project and propose intelligent services that can be used to ease tasks and procedures. Included in these intelligent services are the development of heuristics for optimization, pattern discovery through the analysis of inputs and process optimization by process mining.

The main requirements requested deal with the improvement of the elaboration of Working Instructions (WI) and the planning of Standard Work (SW), accordingly to the Lean Thinking principles and in the context of the Industry 4.0 framework. Work Instructions (WI) and Standard Work Sheets (SWS) are tools that support production and 
play key roles in the engineering process of the organization, impacting performance and the meeting of customer's requirements.

WI provides information about the sequence of operations needed to perform a task correctly by an operators in a workstation. It is provided to operator when its creation is completed and aid the operator with instruction for the manufacturing process. SW is made from the planning of a set of WI that compose a manufacturing line and allow effective line balancing, allocating tasks to operators which are developed in or across workstations. The main problem in SW is task allocation by each operator. The nonexistence of decision support system to aid this planning motivates the development of this project which aims to help analyse target quantities, number of operators and time available for production while reducing errors, alerting users and promoting continuous improvement.

The target company aims to have access to real-time updated information, integrated with other information systems already in use, to support the decision-making process and automatically aid the generation of WI and SW according to a business process.

This article will be organised in four sections to introduce describe the main phases of the work developed. In the production and control processes section a brief overview of existing state of the art contextualized with the target company is made, as well as, an overview of the solution provided by this research. In an ambient intelligencebased decision support system to enhance production and control planning a detailed description of the research results is done, outlining the system architecture, decision support capabilities and the influence and adequacy of ambient intelligence towards this problem. The discussion of results is made in a separate section prior to the conclusion.

\section{The Production and Control Processes}

The production and control processes in large companies demand high complexity, for which technology may help increase performance and competitiveness. One example is the standardization of the supply chain. It is essential to have well defined procedures, trained workers and available resources. This a requirement to work properly, as well as, improve on business processes, increase and adjust the capacity required by the customer and decrease wastes, costs and useless resources[4]. Therefore, the manufacturing process is a crucial phase in the supply chain because it assures that the operations and tasks are performed in controlled and standardized terms, adding to this process a higher value and guaranteeing the same consist quality across manufactured objects [6].

Due to the high complexity of the processes, companies try to standardize every process in its supply chain, in order to achieve a higher performance and competitiveness. It is crucial to have well-defined procedures, so that the processes can be improved.

As presented in the previous section, this work pretend to standardize the fabrication and control processes, using a lean line tool, like Standardized Work, that consists in three components:

- Takt Time - Is the demand rate and serves as a reference to set the pace of production; 
- Work Flow - the sequence in which an operator performs tasks within takt time;

- Standard Inventory - includes units and machines, required to keep the process operating smoothly.

The Standardized Work allows an effective line balancing, allocating tasks to operators and workstations[3]. It is possible to establish an optimized standard work sequence taking into account several constrains, such as the Takt Time, operation time and movements. Additionally, the Standard Work provides relevant information for the manufacturing process that helps the decision-making process.

\subsection{Modelling and Mining the Process}

Process optimization is a beneficial task that most organizations do in order to achieve operational gains and increase the efficiency of their processes. The area for process optimization in the field of information Technologies (IT) may be called process mining (van der Aalst, 2011). The optimization is made through structures such as Work-flow Management (WM) Systems which register the start and end of activities (van der Aalst et al., 2007). Each organization often has log of operations often referred as event log, history or audit trail. A process instance is referred to the task being processed such creation of Work Instruction (WI) or Standard Work (SW) computation. Within each process, there are activities performed and associated with the process instance in these logs. From activities and process logs it is possible to represent process work-flows in graph structures assessing dependencies and where possible. Special analysis on the process is made through the use process mining algorithms in order to optimize each work-flow. A work-flow engine, has the task to automate the tasks within the workflow creating automations for each task or waiting on input to conclude other in order to manage an entire work-flow. These engines suffer limitations due to activities performed that are unaware of the process, therefore they may not be involved in the management and planning of the process by themselves. Systems where each task is aware of its process are called Business Process Management Systems (BPMS) or Process Management Systems (PMS). Either terms define systems that rely on process models to control process work flow. Work-flows contain ordered activities describing their casual dependencies, temporal properties and creation and use of data.

In order to better understand the whole process, it was carried out a process analysis supported by BPMS aimed to map the process and identifying the problems and nonstandardized procedures. Through the analysis of the outcome was possible to identify six main problems that result from the use of non-standard tools:

- Low productivity;

- Low efficiency;

- High time consumption;

- High resources consumption;

- High errors occurrence;

- Orders delays. 
Regarding to the main causes that lead to the effects mentioned, they ranged from human errors (due the lack of training and lack of information of decision-making process), software integration variability, to Work Instruction and SW procedures that require a lot of manual operations and are supported by several non-standard tasks. Moreover, it was pointed out that the process of creation of a Standardized Work usually includes inputs from different departments, under different formats, making this process harder to streamline. Regarding the users, it is emphasized the SW creator and the team leader line, who are responsible to check if the production process occurs accordingly to the predicted SW in the assembly line. From the analysis of the requirements, we concluded that to manage and create a Standardized Worksheet, it is required inputs from several departments and systems:

- the precedence table of the workstations from the testing process (an Excel file);

- an assembly line layout in order to figure out the operators movements (a CAD file);

- some data, like the percentage of machine usage and minutes per shift from the company Intranet (an Excel file);

- a set of tasks from the Work Instructions (an Excel file);

- MTM measurements (a PDF file);

- the product to be produced quantities;

- machine times to calculate the time per shift (an Excel file).

About the SW management process, it involves its creation, its update, its deletion and search possibilities. Eventually, it is important to notify the team leader line that a new SW is ready, as well as receive any comment based on the SW consulted by the team leader line that might change it. The manufacturing and control processes are bounded to use the Standardized Work, since it brings optimization of the procedures, as well as services with higher quality and productivity[5].

\subsection{The Solution Overview}

Presently, the processes of WI and SW creation are supported by Excel files, which require a lot of manual operations and time consumption. Additionally, these are paperbased processes that lead to order delays, low efficiency and productivity. Moreover, changes in WI and SW cannot be visualized in real-time which implies delays and potential errors updating a large database of files, transmitting files across departments or consulting different versions of the same WI and SW. This situation can be improved with an integrated and intelligent Decision Support System (DSS) to enhance productivity, planning efficiency and response capability to an high variability context and uncertain demand by reducing time, resources consumption and errors events. In this sense, there can be stated key purposes that the proposed system addresses:

- Process automation: the procedures must be supported by an system that minimizes the manual data insertion and the human-error;

- Information real-time update: the information flow must be automatic and provided by the system, in order to ensure the data accuracy; 
- WI visualization in displays: paper-based process will be avoided and the WI will be visualized through displays that provide relevant information about the assembly process using animations for a better understanding;

- Support in the decision-making process: During the SW development, there are some constraints that must be fulfilled. the system should be able not only to support the decision-making process, but also through the optimization and the choosing of the best scenarios;

- Provide analytical support: having the access to the manipulation of a time-series of internal historical company data, real-time operational data and sometimes external data the analytical model of the system will provide a highest level of decision support linked to analysis of large collections of historical data and real-time data.

- Applying Ambient Intelligence approaches: a set of AI methods will be available for reasoning under uncertainty, to support complex decision analysis regarding core planning procedures and also learning, in order to adapt in a dynamic environment.

The analysis of both current business process and the functional and non-functional requirements resulted in the conceptualization of use cases for each scenario, WI and SW and potential clients of the Decision Support System (DSS).

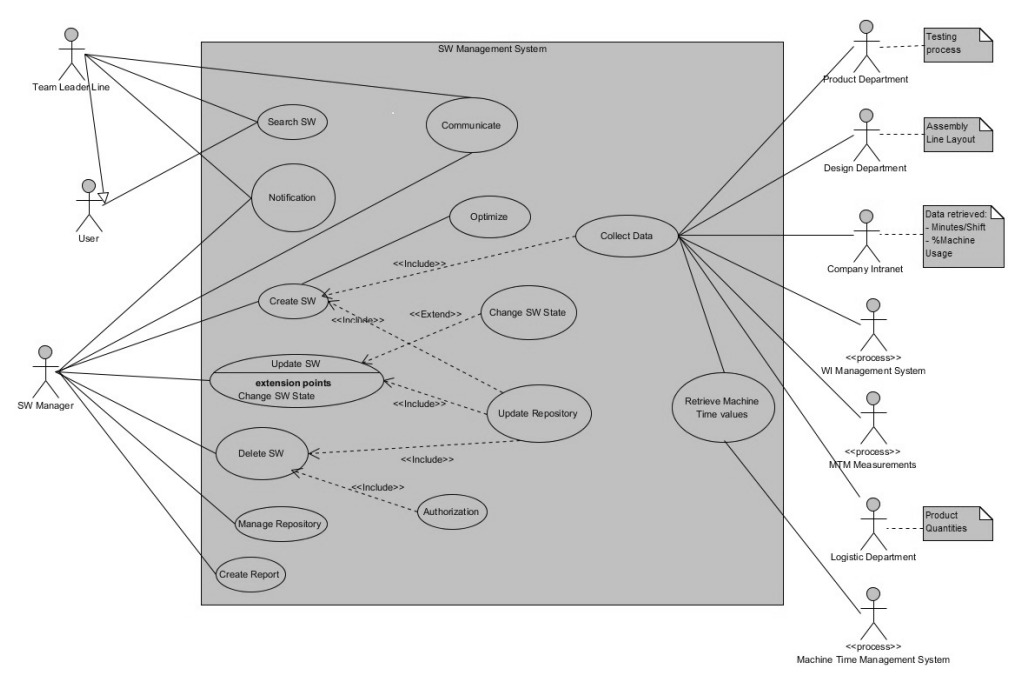

Fig. 1. Work Instructions Use Case

In the case of WI creation, update and availability the use case diagram in picture 1 entails the list of operations required, as well as, datasources and client permissions.

Using the same strategy, the creation, update and availability of SW, in picture 2 details client permissions, datasources and the list of operations required.

Bearing in mind that the current state is characterized by the lack of integration with other IT systems, the proposed system was designed after a detailed analysis of func- 


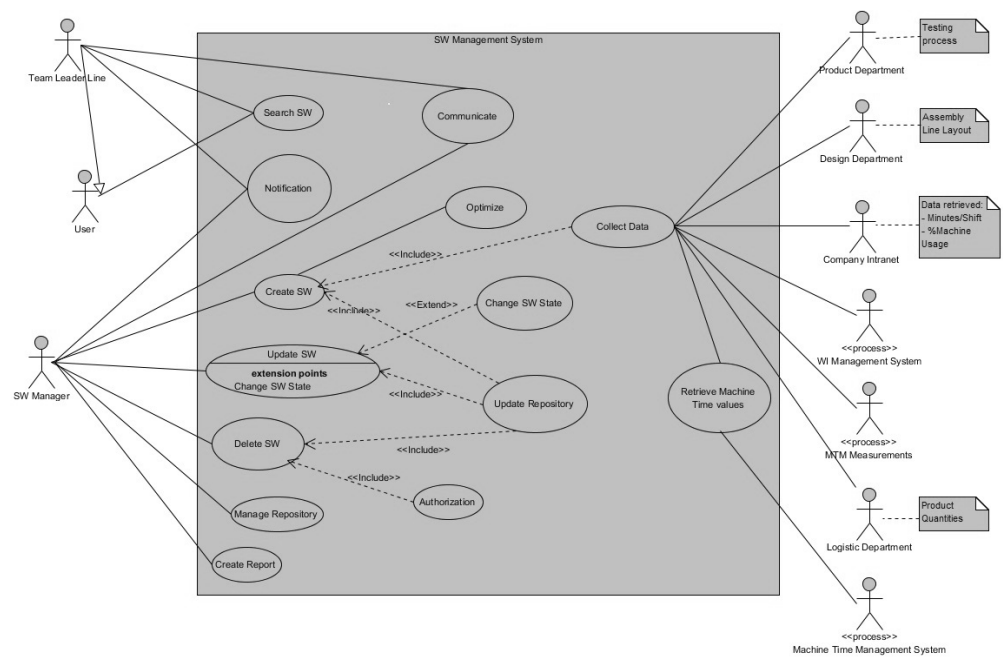

Fig. 2. Standardized Work Use Case

tional and non-functional requirements captured by direct interviews with stakeholders with the objective of circumventing this issue. Moreover, it was also taken into account the business objectives related to the need of improving production outcomes and the decision-making performance by implementing production optimization.

Therefore, an intelligent Decision Support System is proposed to answer these issues and support managers making timely and optimal decisions. These systems are designed specifically to facilitate decision processes. They support rather than automate decision making and are able to respond quickly to changing needs of decision makers. Traditionally, academics and practitioners have discussed building DSS regarding four major components - 1) the user interface, 2) the database, 3) the models and analytical tools, and 4) the DSS architecture and network. This traditional list of components remains useful because it identifies similarities and differences between categories or types of DSS and it can help managers and analysts build new DSS. In the following section, we depict our solution using this approach.

\section{An Ambient Intelligence-Based Decision Support System to Enhance Production and Control Planning}

After the process of requirements specification, we designed an intelligent system comprising several building blocks, including: architecture and networking design, refer to how hardware is organized, how software and data are distributed in the system, and how components of the system are integrated and connected; the model-driven module, emphasize access to and manipulation of a multi-criteria model to provide decision support; and a ambient intelligence module, use artificial intelligence methods to produce relevant knowledge, avoid error and create intelligent services through software agents. 
The objective of the system is to change the actual process, which includes automate parts of the process with information systems, optimize and improve efficiency in the processes, as well as reduce errors. Moreover, it is intended that the system should not only optimize the standard work and work instructions by providing dynamically allocation of tasks, but also provide a tool that assist and support the control of production processes.

\subsection{The System's Architecture}

It is intended that the application, not only should optimize the work instructions in the assembly lines and the standard work, providing dynamically the allocation of tasks, but also should provide a tool that help and support the control and production processes. To achieve these goals, was created the architecture depicted in figure 3. It consists of a Core Server that is composed by four components: Database Handler, Authentication Agent, Standard Worksheet Handler and Standard Worksheet Engine. The Database Handler is responsible to manage the access to the database, containing the constraints of production and control planning, the product information, the work instructions, the table of precedence (which gives the flow between the stations), the tasks to be performed by the operators, etc. On the other side, the Standard Worksheet Handler manages the access to standard worksheets information in Production and Control Planning Repository, and the Authentication component is responsible for authenticating the user into the system. The Standard Worksheet Engine interprets all the control and planning constraints, product information, the work instructions and provide standard worksheets alternatives. This component consists in a set of rules and optimization models that can constrain possible solutions, as well as alternative solutions and methods for evaluating them. These standard worksheets alternatives can be access through RESTful web services in order to ensure they can be easily integrated into any type of application. The Core logic is implemented with web languages in a client server model where information is stored in a central server that provides the information to thin clients through web pages .

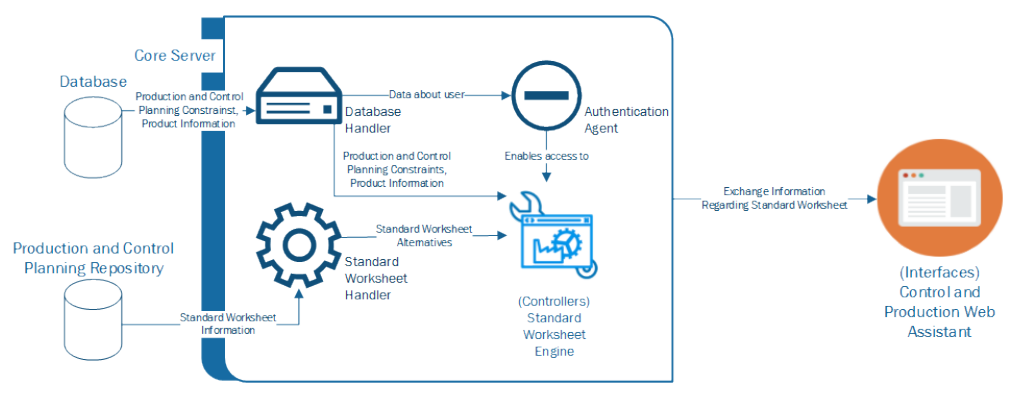

Fig. 3. Architecture of the Production and Control Planning System.

The Control and Production Web Assistant, which uses the web services available in the Core Server, will be develop as a web application following the Model-View- 
Control (MVC) pattern. This assistant provides the standard worksheets alternatives for the user to evaluate them. In order to allow the users interaction, this application offers the possibility of changing any attribute of the standard worksheet and the possibility of recalculate the standard worksheet to get the viable solution. The application also will provide a message box to allow and facilitate the communication between the different participants in this process. These alerts are shown as side messages and are gathered in a notification stack.

\subsection{Model-Driven Representation}

Model (a mathematical representation that relates variables) and analytical models are a dominant component in an intelligent Decision Support-System. When a model is need to understand a situation, then is needed to deliver a representation to the users of the system. In many industrial engineering applications the final decision is based on the evaluation of a number of alternatives in terms of a number of criteria. This problem may become a very difficult one when the criteria are expressed in different units or the pertinent data are difficult to be quantified. The Weighted Sum Model is an effective approach in dealing with this kind of decision problems. Moreover, it is a simple model used to identify and choose alternatives based on the values and preferences of the decision maker. It combines the different objectives and weights corresponding to those objectives to create a single score for each alternative to make them comparable. On the other hand, using a mixed integer linear programming model for the production line balancing and optimization, which will allow to reduce the resources used, the errors introduced by the manual operations, increases automation and human independence process.

We purpose, firstly, to develop a simple mathematical model to support the decisionmaking regarding the manufacturing process. The objective is rather expose the data patterns and relationships to have a automated mean to extract information and knowledge that is useful for decision support. Also this strategy will allow asynchronous processing of the available inputs, provide greater flexibility in modelling, and allow adaptive channel weighting between different conditions [7]. Secondly, to combine the previous model (that provides a basic functionality) with the incorporation of optimization models to analyse complex data. This approach is oriented towards finding the best decision alternative to generate optimal solutions that are consistent with time and resource constraints. Furthermore, we intent to develop a catalogue of different classes of models that seems useful to solve a specific class of problems like a scheduling problem or a combinatorial search problem. The intent is to develop a catalogue of models including all the models that allow an effective line balancing, allocating tasks

to operators and workstations and yield relevant information for decision-making in the manufacturing process.

\subsection{The Ambient Intelligence Approach}

An intelligent environment acts on behalf of its users anticipating needs and easing tasks. Developing projects with this mentality require inputs from environment sensors, 
detection of tasks and actuators. In case of an industrial environments, this vision is kept, though with slight modifications to adequate the norms to a professional space.

An abstract notion of sensors and actuators can be used to include software agents as both sensors and actuators in a information systems approach. These agents obtain relevant data to each process and deliberate actions based on heuristics from artificial intelligence, expert systems and machine learning autonomously on behalf of human collaborators in hope to provide meaningful suggestions, action or plans of action.

In the case of the work proposed in this article, current data is compared to historical data to discern how a process should be run. Analytics provide recommendations, enhancements and warnings to support decision-making. This results in effective, productive, environmentally friendly and cost-effective production.

Experience from past known approaches are used as input in a machine learning algorithm, the ANN, which is trained to learn patterns on the inputs and relate them with known outcomes. After the learning phase the ANN is able to provide answers to inputs on a online system which seeks optimizations and act faster than traditional heuristics which can take, hours, days or weeks to provide the desirable results. This system combines the intelligent environment approach by capturing initial data from the collaborator traditional work, and on later stages agents propose working solutions to the collaborator anticipating his work.

\section{Discussion}

Results in this approach come from the application of a requirement analysis with interviews to the stakeholders of the project and the detailed mapping of requirements and their adoption to the ambient intelligence patterns increasing efficiency and value to the business process. This business process transformation is inspired in the industry 4.0 moto and intelligent manufacturing.

Intelligent services are also provided by software agents that accompany traditional business tasks such the planning of the standard work and provide suggestion based on previous approaches and their results. Such is the case with the ANN detailed in this proposal. More services shall be detailed in future iterations of this project but this first one lays the ground work to acquire a new mentality supported by the concepts of ambient intelligence.

The design used and sensor agent may be useful towards other tasks inside process mining, such as finding recurrent patterns in the standard work design which can evidence strategies to speed up optimization tasks. For instance, discovering that a certain layout in similar standard work provides local optimal results may be used on new similar designs. This similarity may be again calculated by software agents and detailed to the users later.

We aim to provide a proof-of concept application intelligent services and ambient intelligence patterns at the disposal of business processes and how they can be related. This approach successfully demonstrates this strategy and outlines its potential gains in terms of work automation and increase in efficiency. All this then be translated into added value to the manufacturing process which is the desire of the stakeholders. 


\section{Conclusion}

This work details the transformation of a business process, SW and WI planning, inside a manufacturing company. Our approach uses the ambient intelligence, machine learning and multi-agent systems to manage the process flow and en results. It was proposed an ongoing project to design and develop an intelligent decision support system for users to make effective decisions regarding the process of Work Instructions and Standard Work procedures. A vision of the desired solution, as well as, different approaches were provided in their early stages and seeking potential modifications.

The future research should continue to explore the potential improvement of the decision-support system in terms of including non-quantifiable criteria, the meaningful information from additional ambient intelligent services and the ways to link and relate the historical data to new data gathered from a wide range of sources with automatic data feeds. Other opportunities include using the ambient intelligent approach to make autonomous notification of collaborators of new solutions on prolonged tasks such as the ones derived from linear optimizations. In this light, agent may also derive a working solution through machine learning and autonomously search optimal ones using the traditional algorithms, or evolutionary algorithms. In the event of obtaining a better solution it may then autonomously update the plan with the new found solution or alternatively notify the collaborator of the new solution available.

\section{Acknowledgements}

This work has been supported by COMPETE: POCI-01-0145-FEDER-007043 and FCT - Fundação para a Ciência e a Tecnologia (Portuguese Foundation for Science and Technology) within the Project Scope UID/CEC/00319/2013. This research is also sponsored by the Portugal Incentive System for Research and Technological Development. Project in co-promotion $\mathrm{n}^{\mathrm{o}}$ 002814/2015 (iFACTORY 2015-2018).

\section{References}

1. van der Aalst, W.M.P., Reijers, H.A., Weijters, A.J.M.M., van Dongen, B.F., Alves de Medeiros, A.K., Song, M., Verbeek, H.M.W.: Business process mining: An industrial application. Inf. Syst. 32(5), 713-732 (Jul 2007), http://dx.doi.org/10.1016/j.is.2006.05.003

2. van der Aalst, W.M.P.: Process Mining: Discovery, Conformance and Enhancement of Business Processes. Springer Publishing Company, Incorporated, 1st edn. (2011)

3. Emiliani, M.: Standardized work for executive leadership. Leadership \& Organization Development Journal 29(1), 24-46 (2008)

4. TEAM, P.P.D.: Standard work for the shopfloor (2002)

5. Ungan, M.C.: Standardization through process documentation. Business Process Management Journal 12(2), 135-148 (2006)

6. Williams, B.A.: Standard work-lean tools and techniques. Tech. rep., SAE Technical Paper (2001)

7. Wu, L., Oviatt, S.L., Cohen, P.R.: Multimodal integration - a statistical view. IEEE Transactions on Multimedia 1, 334-341 (1999) 